Para enlazar con este artículo / To link to this article:

http://dx.doi.org/10.14198/fem.2019.34.08

Para citar este artículo / To cite this article:

Peral Crespo, Amelia. «Perspectiva de género en la literatura de la Shoá: una escritura transgeneracional». En Feminismo/s, 34 (diciembre 2019): 175-198. Dosier monográfico: Estado actual de la investigación en Literatura francesa y Género: balance y nuevas perspectivas / État présent de la recherche en Littérature française et Genre: bilan et nouvelles perspectives, coord. Ángeles Sirvent Ramos, DOI: 10.14198/fem.2019.34.08

\title{
PERSPECTIVA DE GÉNERO EN \\ LA LITERATURA DE LA SHOÁ: UNA ESCRITURA TRANSGENERACIONAL
}

\author{
HOLOCAUST LITERATURE WITHIN A GENDER \\ PERSPECTIVE ON TRANSGENERATIONAL WRITING
}

\author{
Amelia PERAL CRESPO \\ Universidad de Alicante, Alicante \\ Amelia.Peral@ua.es \\ orcid.org/0000-0002-9923-7619
}

\section{Resumen}

Desde los años 80 hasta la actualidad, los estudios de género han mostrado su interés por desentrañar el papel que desempeñó la mujer judía durante el genocidio nazi. El objetivo de este artículo es, en un primer momento, poner de manifiesto la polémica suscitada en el seno de los estudios de género y del Holocausto a la vez que analizar la literatura de la Shoá desde esta perspectiva, para así, ir adentrándonos de forma más específica en las obras narrativas pertenecientes a la primera generación de la memoria de la Shoá y en las obras narrativas pertenecientes a la segunda generación tras la memoria de la Shoá, y dar respuesta a la pregunta fundamental: ¿podría la literatura transgeneracional de la Shoá ser estudiada desde una perspectiva de género?

Palabras clave: Shoá; escritoras; transgeneracional; memoria; genocidio.

\begin{abstract}
From the 80s to the present, gender studies have examined the role Jewish women have had during the Nazi genocide. The aim of this article is to highlight the controversy raised within gender and Holocaust studies while analyzing the Holocaust
\end{abstract}

Los contenidos de la revista se publican bajo una licencia de Creative Commons Reconocimiento 4.0 Internacional (CC BY 4.0)

Feminismo/s 34, diciembre 2019, pp. 175-198 
literature from this perspective. In order to do this, it is important to focus on the narrative works belonging to the first generation of the memory of the Holocaust as well as on the narrative works belonging to the second generation after the memory of the Holocaust, and giving answers to the fundamental question. Can the Holocaust transgenerational literature be studied from a gender perspective?

Keywords: Holocaust; Writers; Transgenerational; Memory; Genocide.

\section{INTRODUCCIÓN}

Desde los años 80, los estudios de género han mostrado su interés por desentrañar el papel que desempeñó la mujer judía durante el genocidio nazi. ¿Existió una política de exterminio distinta en el caso de que las víctimas fueran mujeres? ¿O tal diferencia no existió y, en este caso, la Shoá no podría ser analizada desde una perspectiva de género?

Esta corriente surgió en Estados Unidos a principio de los 80, cuando los estudios de género y los estudios sobre el Holocausto confluyeron a la hora de abordar la Shoá desde una perspectiva de género.

El objetivo de este artículo es poner de manifiesto la polémica suscitada en el seno de los estudios de género y del Holocausto, que abarca principalmente países como Estados Unidos, Alemania, Israel y Gran Bretaña, pero que tiene poca repercusión en Francia a pesar de algunos intentos. Desde hace ya algunos años, en España va cobrando fuerza esta nueva perspectiva. En un primer momento, nos acercaremos a la polémica entre los estudios de la Shoá ${ }^{y}$ los estudios de género para, posteriormente, analizar la literatura de la Shoá desde una perspectiva de género al adentrarnos en la escritura transgeneracional propia de las segundas generaciones de la memoria, y terceras generaciones tras la memoria de la Shoá.

¿Puede la literatura transgeneracional de la Shoá ser estudiada desde una perspectiva de género? ¿Qué características la definirían?

1. En España al igual que en Francia, prevalece el término «Shoá»por encima del de «Holocausto». El término «Shoá» proviene del hebreo y significa «catástrofe». Comenzó a ser utilizado en Francia tras el estreno en 1985 del documental homónimo de Claude Lanzmann. En Norteamérica, sin embargo, el término más empleado es el de Holocaust.

Feminismo/s 34, diciembre 2019, pp. 175-198 


\section{ACERCAMIENTO A LOS ESTUDIOS DE LA SHOÁ DESDE UNA PERSPECTIVA DE GÉNERO}

En Estados Unidos, a principio de los años 80, existió una corriente dentro de los estudios de género que comenzó a interesarse por la suerte que las mujeres judías habían corrido durante la persecución nazi. Sin embargo, tal y como apunta Isabelle Ernot en su magnífico trabajo, publicado en 2015, tres décadas han pasado y sólo existe una quincena de obras colectivas e interdisciplinares, publicadas básicamente en lengua inglesa entre los años 1984 y 2014. La mayoría de esas publicaciones están realizadas por investigadoras que se interesaron también por las segundas generaciones.

En los años 80, tres son las historiadoras americanas que destacan: Renate Bridenthal, Atina Grossman y Marion Kaplan, especialistas en el nazismo. Editan la publicación When Biology became Destiny. Women in Weimar and Nazi Germany (1984), en la que participan otras historiadoras como Claudia Koonz, Sybil Milton, Gisela Bock y Karin Hausen. Analizan de forma específica la condición de las mujeres durante la Alemania nazi.

En 1983, Esther Katz y Joan Ringelheim publican Proceedings of the Conference: Women Surviving the Holocaust. Desde una perspectiva histórica, este ensayo supone un hito fundamental en las investigaciones de género sobre la Shoá, puesto que es la primera vez que se plantea la necesidad de estudiar la vida de las mujeres judías deportadas y asesinadas.

En 1985, Joan Ringelheim publica un artículo «Women and the Holocaust: A Reconsideration of Research». De dicha publicación se desprenden varios temas, tal y como señala Ernot, que ocuparán las investigaciones sobre los estudios sobre el Holocausto, desde una perspectiva de género, a lo largo de la década de los 80 y hasta la actualidad:

Deux priorités y sont définies: mettre en lumière le vécu des persécutions subies par les femmes, les modalités de survivance dans les ghettos et les camps, ainsi que leurs attitudes résistantes et, dans une optique plus générale, travailler pour que l'histoire des femmes contribue au savoir sur l'Holocauste (Ernot 3).

En este sentido, los estudios de género desarrollan dos ejes temáticos haciendo hincapié en las mujeres y en el papel que desempeñaron durante la Alemania 
nazi, y con respecto a las mujeres judías sobre su papel de víctimas durante el nazismo.

Ringelheim fue pionera, en la década de los 80, a la hora de destacar el escaso papel acordado a los testimonios de mujeres frente a los testimonios masculinos ${ }^{2}$ ya indiscutibles, como los de Primo Levi (1947), Elie Wiesel (1958), Piotr Rawicz (1961).

Tras la década de los 80 cobran fuerza los trabajos conjuntos realizados por investigadoras americanas e israelíes. Las primeras conferencias en Israel fueron organizadas en 1994-1995 por Dalia Ofer, coeditora junto a Leonor Weitzman, en 1998, de Women and the Holocaust. Ofer y Weitzman presentaron un interesante estudio, traducido al francés y publicado en 2008, «Sur la spécificité de la place des femmes dans la Shoah. Fondements théoriques d'une analyse». Las autoras plantean desde el inicio de la investigación su objetivo principal:

Notre propos n'est pas de prétendre que le vécu des femmes pendant la Shoah fut entièrement différent de celui des hommes; mais il serait tout aussi erroné et fallacieux d'affirmer qu'il fut identique à celui des hommes. Dans bien des cas, l'épreuve d'une personne fut déterminée par son sexe et ce n'est qu'en comprenant ce qui fut spécifique aux femmes - comme ce qui fut propre aux hommes - qu'on peut présenter un tableau complet de la réalité (480).

A partir del año 2000, en Estados Unidos también se realizan algunas tesis que tienen como objetivo principal destacar el papel que desempeñaron las mujeres durante la Shoá, tal y como apunta Isabelle Ernot haciendo referencia a una publicación de Sarah Cushman y Na'ama Shik de 2008: «Memory, Body, Gender: The Female Experience According to Autobiographies Written between 1946 and 2000 by Women Survivors of Auschwitz-Birkenau» (Ernot 8), pero son relativamente pocas. Es el caso de Sarah Cushman, actualmente directora de The Holocaust Educational Foundation of Northwestern University, cuya tesis The Women of Birkenau (2010) analiza desde una

2. Otra cuestión importante es destacar que algunos de esos testimonios también tuvieron que superar otras barreras a la hora de ser editados. Pues tras la guerra, Francia y otros países no estaban preparados para leer ni escuchar testimonios sobre una guerra que el mundo quería olvidar.

Feminismo/s 34, diciembre 2019, pp. 175-198 
perspectiva histórica y social a las mujeres que fueron deportadas al campo de exterminio de Auschwitz.

Durante la década de 2010, es importante citar el reciente estudio que Mirna Goldenberg publicó: Before All Memory is Lost: Women's Voices from the Holocaust. Por medio de relatos en prosa, diarios o poemas, veinticinco mujeres, supervivientes de la Shoá, reflexionan sobre las experiencias vividas. Ese mismo año, Zoe Waxman lanzaba el siguiente estudio: Women in the Holocaust: A Feminist History, consciente de que, a pesar de los años transcurridos desde que los estudios sobre el Holocausto y los Estudios de Género comenzaron a debatir sobre las similitudes y diferencias entre mujeres y hombres durante el exterminio judío, sigue siendo necesario escuchar las voces de mujeres que han podido contar la experiencia traumática vivida. Actualmente, las y los historiadores, como afirma Waxman, siguen debatiendo sobre la existencia de una política de exterminio específica en función del sexo.

En Europa, durante los primeros años del nuevo milenio, investigadoras como Bárbara Distel y Gisela Bock se centran en el estudio de las mujeres judías durante el genocidio. Es el caso de la alemana Barbara Distel, directora del Memorial del campo de concentración de Dachau, y cuya publicación, en 2001, de Frauen im Holocaust, ofrece un acercamiento a la historia de la Shoá desde el mundo de la mujer. También contribuyó, junto a Wolfgang Benz, a los volúmenes Der Ort des terrors. En 2006, el volumen 4 dedica un número especial al campo de Ravensbrück. Ravensbrück fue hasta junio de 1941 el único campo de concentración para mujeres. Y también es el caso de la historiadora feminista alemana, Gisela Bock, cuyo ensayo Genozid und Geschlecht. Jüdische Frauen im nationalsozialistischen Lagersystem, publicado en 2005, se adentra por primera vez, al ser escrito en lengua alemana, en la vida y muerte de las mujeres judías en los campos del régimen nazi.

En Francia, durante los años 90, la socióloga francesa Liliane Kandel publica «Une pensée empêchée: des usages du «genre », et de quelques-unes de ses limites». Se trata de un estudio en el que defiende la no diferenciación por cuestiones de sexo del sistema de exterminio nazi. Así pues, todo va a depender de cómo sean vividas, por los hombres y por las mujeres, las experiencias traumáticas. Unos años más tarde, contribuye con «Sur quelques lectures féministes du nazisme» a la publicación 1939-1945: combats de femmes. Françaises et Allemandes, les oubliées de l'histoire. Con esta contribución quiere

Feminismo/s 34, diciembre 2019, pp. 175-198 
poner de manifiesto la escasa repercusión que han tenido las mujeres en Francia, durante la Segunda Guerra Mundial, al menos hasta principios del nuevo siglo, con respecto a los estudios que se han ido llevando a cabo en Estados Unidos e incluso en Alemania.

En 2004, dirige la obra colectiva Féminisme et nazisme, que analiza el papel desempeñado por las mujeres durante el nazismo. Algunas veces han sido tildadas de heroínas, otras veces han sido consideradas culpables, víctimas o resistentes, pero siempre con una voz que ha querido ser silenciada. En el estudio que presenta en esta obra, «Femmes, féminismes, nazisme, ou: on ne naît pas innocent(e), on le devient », en el que reescribe, desde el título del mismo, la mítica frase de Simone de Beauvoir «On ne naît pas femme, on le devient », Liliane Kandel propone desde una perspectiva histórica un estudio sobre el papel de las mujeres del III Reich no excluyendo a las mujeres de su implicación tanto directa como indirecta en las políticas nazis:

Deux points se dégagent de ce premier ensemble de textes. Le fait tout d'abord que les femmes, comme les hommes, participèrent aux diverses facettes du système nazi, qu'elles lui apportèrent leur soutient tantôt direct et explicite, tantôt indirect, et que les féministes, pour nombre d'entre elles, n'en furent pas exemptes (11-12).

Kandel menciona el seminario que la historiadora Rita Thalmann ${ }^{3}$ celebró en 1984, en la Universidad Paris 7-Denis Diderot, bajo el título de «Sexe et race» ${ }^{4}$, como uno de los primeros lugares de encuentro, en Francia, donde pudieron intercambiar distintas visiones sobre el papel de las mujeres durante ese periodo. En algunos casos, confiesa que las preguntas incluso insólitas que se hacían entre ellas, como por ejemplo las concernientes a las mujeres fascistas italianas, o falangistas o mujeres nazis (Kandel, Féminismes et nazisme

3. Nacida en Nuremberg en 1926 y fallecida en Francia en 2013, Rita Thalmann fue una historiadora francesa de origen judío. Su padre fue deportado y murió en Auschwitz, y su madre fue ingresada en un hospital psiquiátrico donde falleció. En 1982 publicó Etre femme sous le III ${ }^{e}$ Reich.

4. El seminario surge en un momento determinante en Francia cuando la historia sobre las mujeres se encontraba en pleno auge. De ahí el papel innovador que juega el seminario «Sexe et race», donde se analiza la historia de las mujeres y los movimientos feministas. Un año más tarde, en 1985, crea en la universidad Paris 7-Denis Diderot un centro de estudios y de investigaciones germánicas, posteriormente llamado CERIC, Centre d'Etudes et de Recherches Intereuropéennes Contemporaines.

Feminismo/s 34, diciembre 2019, pp. 175-198 
9) solo podían tener cabida en un espacio como el que ofrecía el seminario de Thalmann. Rita Thalmann había publicado, en 1982, un libro de obligada consulta, Être femme sous le III $^{e}$ Reich $^{5}$. Sin embargo, no podemos obviar su publicación más intimista, la autobiografía que vio la luz en 2004, Tout commença à Nuremberg, cuyo subtítulo es «Entre histoire et mémoire», y que la historiadora no pudo no haber escrito, tras perder a su padre en Auschwitz y ver cómo su madre se iba consumiendo hasta la muerte en un hospital psiquiátrico francés. Thalmann dio rienda suelta a la necesidad de expresar por medio del relato, es decir por medio de la escritura, al proceso de resiliencia que ya se había iniciado muchos años atrás, cuando siguió el camino que la llevaría a ser una de las mayores especialistas francesas sobre el nazismo, el mundo germánico y las mujeres.

Durante los años 90 y principios del nuevo milenio siguen teniendo relevancia, en Francia, los debates que llevan a la no diferenciación entre hombres y mujeres durante la Shoá, aunque van cobrando fuerza algunas ideas en las que se afirma que las mujeres por su condición de mujer tienen mayor resistencia, y que, en ellas, el concepto de «solidaridad» está mucho más arraigado. En este sentido, queremos señalar que estas ideas siguen siendo de plena actualidad. Prueba de ello la podemos hallar en la literatura francesa de la Shoá y en los testimonios de los supervivientes. En la entrevista que Ida Grinspan ${ }^{6}$ nos concedió el 30 de noviembre de 2010 incide sobre ello:

I.G.- si vuelve a leer todo lo que las mujeres han escrito, las mujeres resistentes, ya sea Germaine Tillion [...] Charlotte Delbo, ya sea Geniève de Gaulle. Todas esas mujeres cuando hablan de su experiencia en los campos siempre resaltarán la solidaridad. Y ésta aparece mucho menos en los hombres (Peral, Memoria de la Shoá. Literatura y testimonio 283).

I.G.- la solidaridad material para nosotras no existió, pero sí existió una solidaridad física [...] Dos formas de solidaridad: física y moral. Material, no teníamos nada. No se puede compartir lo que no se tiene (Peral, Memoria de la Shoá. Literatura y testimonio 291).

5. A raíz de este libro, entabló una polémica con la historiadora alemana Gisela Bock.

6. La mencionaremos de manera más detallada en el siguiente apartado. 
Y como contrapunto a las declaraciones de Ida Grinspan, el testimonio que Víctor Pérahia ${ }^{7}$ nos concedió el 3 de diciembre de 2010 también es revelador en ese sentido:
V.P.- No existía entre los hombres [...] El único instinto que nos mantenía con vida era el instinto de conservación. Intentábamos escapar de ello, pero la solidaridad, la amistad, los sentimientos de unos hacia otros, era de cada uno hacia sí mismo. Te daban raciones ínfimas y cuando te las dejabas [...] te las robaban. Eso no era solidaridad (Peral, Memoria de la Shoá. Literatura $y$ testimonio 325$)$.

Tampoco podemos destacar, en Francia, un gran número de tesis que tengan como objeto de estudio la perspectiva de género. Cabe mencionar, en ese sentido, la tesis de Jennifer Cazenave, defendida en 2011, en la universidad de Paris 7, bajo el título Genèses des figurations de la femme dans la Shoah: voix féminines et représentations de l'Holocauste (1946-1985).

Habría que destacar también el reciente estudio de Ernot, ya mencionado al comienzo de este apartado, pues aporta en el anexo unos datos estadísticos interesantes sobre la deportación entre hombres y mujeres desde Francia y su posterior asesinato en Auschwitz-Birkenau, en el periodo comprendido entre $1942^{8}$ y 1944 , datos que ponen en entredicho los debates suscitados durante los años 80, 90 y principios del nuevo milenio en cuanto a la no diferenciación entre hombres y mujeres durante la Shoá. Para ello tiene en cuenta los siguientes parámetros:

1. Deportación hacia Auschwitz-Birkenau

2. Gaseadas/os al llegar

En 1942, sobre 41958 deportaciones, 57\% corresponderían a hombres y 42,2\% lo serían de mujeres. De entre todas las deportaciones, $26 \%$ de los hombres fueron gaseados al llegar y $28 \%$ de las mujeres lo fueron también al llegar.

7. Fue detenido en 1942 y liberado en 1945. Pasó por distintos campos de concentración, entre ellos el de Bergen-Belsen.

8. El campo de concentración y exterminio de Auschwitz-Birkenau empezó a admitir mujeres en la primavera de 1942. Antes solo era un campo para hombres: «Les choses changent le 26 mars 1942 avec l'arrivée le même jour de 999 femmes juives de Propad en Slovaquie et d'autant de femmes allemandes de Ravensbrück -prisonnières politiques » (Wieviorka 75).

Feminismo/s 34, diciembre 2019, pp. 175-198 
En 1943, de 13066 deportaciones, 53\% son relativos a hombres mientras que el $46 \%$ corresponden a mujeres; $30 \%$ de los hombres fueron asesinados al llegar frente al 35\% de las mujeres.

En 1944, sobre las 14143 deportaciones, el 52,2\% pertenecen a hombres y el $47,5 \%$ a mujeres; el 34\% de los hombres fueron ejecutados al llegar y el $36 \%$ de las mujeres también.

Pour les trois années, le nombre total des personnes assassinées à l'arrivée par gazage, indique une différence entre hommes et femmes (par rapport à leur groupe sexué) : $52 \%$ de tous les hommes et $70 \%$ de toutes les femmes, soit un homme sur deux et deux femmes sur trois (Ernot 17).

Estos datos ponen de manifiesto que pudo existir una diferencia frente a lo que se pensaba hace años. Además es importante mencionar que Ernot presenta un estudio sobre el campo de exterminio de Auschwitz y que, hasta 1941, sólo existió un campo de concentración para las mujeres, en Ravensbruck. En los otros campos de concentración y de exterminio, las mujeres estaban separadas de los hombres.

En España, la situación es similar a la francesa, pero desde finales del año 2000, hay una línea de investigación ${ }^{9}$ que se ha dedicado de forma específica a estudiar, desde una perspectiva literaria, los textos de escritoras de la Shoá de expresión francesa.

Para concluir este primer apartado en el que hemos querido ofrecer una breve aproximación a las confluencias entre los estudios sobre el Holocausto o Shoá y los estudios de género desde los años 80 hasta la actualidad, es necesario señalar que los estudios sobre el Holocausto se desarrollaron de manera

9. El grupo de investigación de la universidad de Alicante, Midel (Memoria e Identidades Literarias y Culturales) (Vigrob 271) https://web.ua.es/es/grupo-memoria-identidades-midel/presentacion.html ha organizado diversos seminarios desde 2009 hasta la actualidad sobre dicho tema. Es el caso de los siguientes seminarios: I Seminario Internacional «Escrituras y Voces del Holocausto» (2009); Seminario Internacional «La Shoá desde la memoria y la literatura: nuevas aproximaciones, nuevas expectativas» (2012); Seminario Internacional e Interdisciplinar «Memoria, trauma e identidad» (2013); Seminario Internacional e Interdisciplinar «La Shoá y los Juicios de Nuremberg: víctimas, verdugos, mediadoras y mediadores» (2017); Seminario Internacional: «Memoria, escritura y resiliencia» (2018). También ha publicado distintos ensayos: Arráez, José Luis y Amelia Peral (eds) (2012), Camarero, Jesús (eds) (2015).

Feminismo/s 34, diciembre 2019, pp. 175-198 
diferente en cada país, al igual que los estudios de género y que, por lo tanto, las confluencias entre ambos, así como sus diferencias, han dependido del país de procedencia. No olvidemos que, tras la guerra, la mayoría de los países no estaban preparados para enfrentarse a un genocidio ${ }^{10}$ como el que se había perpetrado contra el pueblo judío. Actualmente, los estudios se centran en la cuestión del cuerpo femenino en relación con la figura materna ${ }^{11}$. Para ello, cada vez es más y más necesario, analizar los testimonios desde la literatura y con una perspectiva de género como se pone de manifiesto por medio de la escritura transgeneracional.

\section{HACIA UNA PERIODIZACIÓN DE LA LITERATURA DE LA SHOÁ DESDE UNA PERSPECTIVA DE GÉNERO}

Durante los últimos casi 10 años, varias han sido las preguntas que se han ido planteando para intentar dar respuesta a la importancia que tiene, cada vez más, la escritura transgeneracional en la literatura de la Shoá. Los testimonios en primera persona son numerosos. Gracias al trabajo realizado por los museos de la memoria como el Memorial de la Shoah en París, la institución israelí Yad Vashem, The World Holocaust Remembrance Center, el Memorial a la muerte de los judíos en Berlín, el Museo del Holocausto en Washington USHM y muchos más..., cada vez tenemos más acceso a ellos. Sin embargo, la realidad nos lleva a otro terreno, doloroso pero inevitable, la progresiva desaparición de esos testimonios en primera persona. ¿Qué quedará tras su pérdida? ¿Qué legado dejarán para las generaciones venideras? Esas preguntas siempre han estado en el centro de sus relatos. Conscientes de su progresiva desaparición ${ }^{12}$, las supervivientes correspondientes a la primera generación de la memoria de la Shoá dieron muestra de la experiencia traumática vivida,

10. La palabra genocidio no aparece en el juicio del 1 de octubre de 1946: «mais on le retrouve mentionné dans les procès suivants. Parce qu'il vise un groupe tout entier, il y est défini comme «une forme aggravée de crime contre l'humanité» (Bensoussan 113).

11. Ernot también hace hincapié en este aspecto en su artículo (14).

12. Únicamente mencionamos a las supervivientes francesas que fallecieron recientemente y que han tenido una gran repercusión mediática por su labor, ya sea como escritoras, cineastas, políticas o que han dedicado parte de su vida en compartir su testimonio.

Feminismo/s 34, diciembre 2019, pp. 175-198 
pero son conscientes de su efimeridad; es el caso de Simone Veil ${ }^{13}$ fallecida en 2017, de Ida Grinspan ${ }^{14}$ en septiembre del 2018, y de Marceline LoridanIvens ${ }^{15}$ también desaparecida en septiembre de 2018.

¿Qué impulsa el ser a escribir? ¿Qué le mueve a intentar decir lo indecible, lo inenarrable? ¿A hablar sobre Ça, uno de los pronombres franceses más utilizados por la mayoría de los y las supervivientes? ¿En qué medida la escritura se convierte en un legado necesario para poder romper el muro de lo indecible? ¿Puede existir una resiliencia? ¿Qué diferencia a las primeras, de las segundas y terceras generaciones e incluso ya cuartas generaciones de la memoria? ¿Y qué papel desempeña la figura materna como transmisora de la memoria olvidada en la reconstrucción del Yo que se escribe en sus textos? Estas preguntas nos han llevado a lo largo de esta década a seguir investigando sobre la importancia que, desde una perspectiva de género, reviste la escritura transgeneracional en la literatura francesa de la Shoá.

Las confluencias entre los estudios sobre el Holocausto y los estudios de género, en Francia, han suscitado numerosas polémicas que han puesto de manifiesto la importancia de estudiar la historia de la Shoá, pero a través de los textos literarios. Llegados a este punto, es importante señalar el ensayo publicado, en 2007, por Anny Dayan Rosenman, Les alphabets de la Shoah, con el que la autora se adentra de manera específica en la literatura de la Shoá, y reflexiona sobre el proceso de escritura que permite salir del silencio. Desde hace ya más de una década, la literatura de la Shoá ha pasado a ser una literatura específica, es decir uno de los temas de estudio de la literatura francesa

13. Nacida en 1927, fue ministra de sanidad y una gran luchadora de los derechos de las mujeres despenalizando el aborto con la célebre «Loi Veil». Actualmente, está enterrada junto a su marido en el Panteón, en París.

14. Nació el 19 de noviembre de 1929 en París de padres polacos de origen judío. Sus padres fueron deportados y asesinados en Auschwitz. En 1944, Ida Grinspan fue deportada también à Auschwitz. Sobrevivió. Narró su experiencia en J'ai pas pleuré en 2002, en colaboración con Bertrand Poirot-Delpech, miembro del Académie Française. En 2012, la editorial Anthropos procedió a la traducción y publicación de su relato.

15. Cineasta y escritora, nació en 1928. Fue deportada con su padre a la edad de 15 años a Auschwitz, Bergen-Belsen y Theresienstadt. Sobrevivió. Es autora de numerosos libros en los que recoge su experiencia traumática: Ma vie balagan (2008); Et tu n'es pas revenu (2015), traducido ese mismo año, Y tú no regresaste; Lamour après (2017). Entre sus numerosas películas cabe destacar: La petite prairie aux bouleaux en 2003 , estrenada en España bajo el título de La sombra de la sospecha.

Feminismo/s 34, diciembre 2019, pp. 175-198 
mostrando de esta manera la necesidad de adentrarse en la literatura de los campos $^{16}$. Sin duda, el otro debate existente en los estudios del Holocausto que ha llevado a cuestionarse si existe como tal una literatura de la Shoá, nos lleva a defender la tesis de Rachel Ertel cuando afirma que:

La question de savoir si elle appartient à la littérature générale me semble sans objet. Appartenir à la littérature n'est en rien contradictoire avec son appartenance à la littérature dite générale. La littérature de la Shoah soulève dans une esthétique qui a ses caractéristiques propres, comme tous les genres littéraires, les questions de l'humain et du déshumain qui sont celles de la fiction, de la poésie et des essais en général (Ertel 214).

$\mathrm{Y}$, es preciso recordar, como apuntamos en el apartado anterior que, tras la liberación de los campos y el progresivo regreso de las y los supervivientes, la realidad a la que tuvieron que hacer frente, como así lo recogen los múltiples testimonios al respecto, es la de enfrentarse a una sociedad que quería olvidar. El silencio se impuso pues, como una medida de supervivencia. Fue un silencio largo y doloroso que marcó a las primeras y segundas generaciones, y que también encontramos en las terceras generaciones. Para la mayoría de los supervivientes, la vuelta a la vida tras los campos fue difícil, pues hablar de lo sucedido no era tarea fácil. El testimonio de Víctor Pérahia es el de un niño que fue detenido junto a su madre. Tras su liberación, tardó 60 años en poder hablar de lo sucedido y en su caso, primero fue la escritura y luego llegó la palabra oral. Como apuntó el propio Pérahia: «no podía hablar de ello porque no encontraba las palabras para decirlo y porque decía que no hay palabras suficientes para contar esta historia» (Peral Memoria de la Shoa. Literatura y testimonio 317).

La historiadora Annette Wieviorka señala que, en 1961, y tras el proceso Eichmann, la palabra de las y los supervivientes comienza a ser escuchada: «Les ouvrages peinaient à trouver des éditeurs et quand ils étaient publiés, ne trouvaient guère de public» (Wieviorka, Les alphabets de la Shoah 8).

Varios intentos de periodización en la formación y evolución de los testimonios orales y escritos se han propuesto a lo largo de la última década. Señalaremos los más relevantes desde distintas perspectivas: psicoanalítica,

16. Hacemos referencia al capítulo 3 de la segunda parte del manual de literatura de Dominique Viart et Bruno Vercier (2005). 
antropológica y sociológica, literaria y filosófica. En 2003, la psicoanalista Régine Waintrater distingue principalmente tres grandes periodos:

a. Un primer tiempo marcado por los testimonios inmediatos tras la postguerra

b. Un segundo tiempo caracterizado por relatos literarios que se publican diez años tras la liberación.

c. Y un tercer tiempo que abarcaría el periodo de 1990 hasta el año 2003, caracterizado por diversas formas tanto literarias como cinematográficas, donde destacan los ensayos y los testimonios que reflejan una clara necesidad de contar y compartir con la sociedad una experiencia que sigue siendo difícil de entender.

En 2007, la antropóloga y socióloga Nicole Lapierre (475-477) propone una periodización en cuatro tiempos.

a. el tiempo de silencio que se inicia inmediatamente tras la guerra y termina 25 o 30 años tras su finalización.

b. el tiempo de la palabra y de la escucha que va de los años 70 al año 85.

c. el tiempo del reconocimiento que comienza en 1985.

d. el tiempo de la globalización, coincidiendo con el inicio del nuevo milenio y con la proclamación por parte de la ONU en 2005, del 27 de enero como día internacional de memoria de las víctimas del Holocausto.

En 2012, el profesor de literatura Alexander Prstojevic (20-28) propone una nueva periodización coincidiendo con los tres periodos señalados por Waintrater, aunque distinguiéndose de estos.

a. La primera etapa va desde el final de la guerra hasta los años 60 .

b. La segunda etapa se inicia con el proceso Eichmann y acaba al final de los años 70.

c. La tercera etapa se centra en los escritores de las generaciones de la postguerra.

El estudio propuesto por el filósofo François Azouvi en 2012, aumentado y reeditado en 2015, sirve de contrapunto a todos los estudios de la Shoá que 
se habían realizado en Francia sobre el silencio, pues desde el principio refuta la tesis del gran silencio sobre la que se ha basado la historia del genocidio. Para ello, basa su estudio en tres grandes partes cronológicas:

a. El genocidio en la cultura francesa desde septiembre de 1944 hasta noviembre de 1961.

b. El genocidio en el espacio público de 1961 hasta 1967.

c. El genocidio en la esfera del Estado de 1971 (Indulto a Touvier ${ }^{17}$ ) hasta el proceso de Klaus Barbie ${ }^{18}$ (1987).

Azouvi demuestra que la salida del silencio se produce de manera lenta y progresiva desde las capas más altas de la sociedad francesa hasta alcanzar el espacio público. Sin embargo, su estudio no se adentra en cuestiones que son fundamentales para comprender a la víctima, es decir, el trauma. A partir de finales de los 90 hasta la actualidad, el concepto de trauma rige la mayor parte de los estudios sobre la Shoá.

Estos intentos de periodización ponen de manifiesto una de las características que más influyen en la literatura de la Shoá, tanto de la primera generación como de las generaciones sucesivas: el silencio. Sobreponerse al silencio. Salir del silencio. Asimismo, estas cuatro propuestas no distinguen entre los escritos de hombres o de mujeres. Waintrater, como psicoanalista, se basa en testimonios, en la mayoría de los casos orales, relatos no literarios. Dedica un capítulo de su ensayo Sortir du génocide a la literatura testimonial, donde plantea el dilema al que todo escritor o escritora tuvo que enfrentarse tras el regreso: escribir, y en este caso responder a la eterna pregunta formulada por el filósofo Adorno, «Peut-on phraser après Auschwitz?». ¿Acaso la literatura, ese lugar de la eterna ficción, podría atreverse a escribir sobre el horror? O si decide no hacerlo, no salir nunca del silencio. Para ello, Régine Waintrater toma como ejemplos escritores ya clásicos dentro de la literatura

17. Touvier colaboró con el régimen de Vichy durante la segunda guerra mundial. Fue condenado a muerte por los numerosos crímenes cometidos. Fue indultado en 1971 por el Presidente Pompidou, y posteriormente, en 1994, encarcelado. Fue el primer francés condenado a cadena perpetua por crímenes contra la humanidad.

18. Fue un militar nazi, conocido como «el carnicero de Lyon» condenado a cadena perpetua por crímenes contra la humanidad. Su proceso, en 1987, tuvo una gran repercusión en la sociedad francesa.

Feminismo/s 34, diciembre 2019, pp. 175-198 
de la Shoá. Es el caso de Primo Levi, Elie Wiesel, Robert Antelme pero también menciona a dos escritoras: Sarah Kofman y Anna Langfus, sobre las que nos vamos a detener. Sarah Kofman, filósofa, conocida por sus ensayos filosóficos, escribió una única obra literaria, su autobiografía y su legado para la posteridad, Rue Ordener, Rue Labat publicada en 1994. Unos meses más tarde, el 15 de octubre de ese mismo año, se suicidó, como también lo hicieron Primo Levi, Paul Celan, André Gorz. Sarah Kofman tenía 60 años y había pasado una parte de su vida intentando dar respuesta a la eterna pregunta que caracteriza la literatura transgeneracional de la Shoá: ¿Qué heredamos de nuestros padres? Para Kofman, que solo tenía 8 años cuando su padre fue detenido en su presencia, deportado, asesinado en Auschwitz y al que nunca más volvió a ver, el inicio de su autobiografía es la respuesta a esa pregunta: «De lui, il me reste seulement le stylo» (Kofman, Rue Ordener, Rue Labat 9). Sarah Kofman fue «une enfant cachée», viviendo con una madre de acogida hasta el regreso de su madre biológica, tras la guerra.

Anna Langfus nació en 1920 en Lublín. Durante la segunda guerra mundial, fue detenida y torturada por la Gestapo. En 1946, llega a París donde permanecerá hasta su muerte en 1966. Su carrera literaria es corta pero intensa. Escribió tres novelas sobre la Shoá: Le sel et le soufre (1960), Les bagages de sable (1962) y Saute Barbara (1965); y cuatro obras teatrales: Les Lépreux (1956), La Récompense (1961) y Amos ou les fausses expériences (1963). La primera, Les Lépreux, es considerada la primera obra teatral sobre la Shoá. Como afirma Jean-Yves Potel, en Les disparitions d'Anna Langfus, fue una de las primeras escritoras en utilizar la ficción para hablar de la Shoá. Para Anna Langfus, la ficción había sido una elección que le ayudó a contar la verdad pues de otro modo no lo habría logrado. Así lo subraya Potel: «La fiction et la construction littéraire, lui ont permis de transmettre, de communiquer un vécu intime : souffrance, désespoir, abandon, peur» (1).

A partir de estas cuatro periodizaciones ${ }^{19}$ y tomando como referencia la propuesta de Régine Waintraiter, proponemos establecer la siguiente

19. Una primera clasificación, tomando como punto de referencia la periodización establecida por Waintrater, se esbozó en 2012 (Peral, «Au-delà des mères perdues»). Se amplió posteriormente en «Le silence, ce cri qui résonne dans l'écriture de Viviane Forrester» (Peral), y actualmente se desarrolla en esta investigación aplicada a la literatura de la Shoá desde una perspectiva de género.

Feminismo/s 34, diciembre 2019, pp. 175-198 
clasificación de las obras narrativas de las escritoras de la Shoá ${ }^{20}$ pertenecientes a las primeras generaciones de la memoria de la Shoá ${ }^{11}$ y a las generaciones posteriores tras la memoria de la Shoá ${ }^{22}$.

1. Testimonios inmediatos tras el final de la guerra: Une Française juive est revenue (1945) de Suzanne Birnbaum; La vie des internés (1945) de Julie Crémieux; Relais des Errants (1945) de Denise Aimé; Sans Armes et sans bagages (1947) de Louise Alcan; Sans Fleurs ni couronnes (1947) de Odette Elina.

2. Testimonios publicados 10 años tras el final de la guerra hasta 1989: la trilogía de Anna Langfus: Le sel et le soufre (1960), Les bagages de sable (1962) y Saute Barbara (1965); Le convoi du 24 janvier (1964) de Charlotte Delbo y los tres tomos Auschwitz et après: tomo I Aucun de nous ne reviendra (1970), tomo II Une connaissance inutile (1970) y tomo III Mesure de nos jours (1971); Ainsi des exilés (1970), L’œil de la nuit (1987), ambos de Vivianne Forrester.

3. Testimonios publicados a partir de 1990 hasta la actualidad ${ }^{23}$ : Ce soir, après la guerre (1992) de Vivianne Forrester; Le mirador (1992) de Elisabeth Gille; Rue Ordener, Rue Labat (1994) de Sarah Kofman; La mémoire et les jours (1995) de Charlotte Delbo; Une petite fille privilégiée. Une enfant dans le monde des camps 1942-1945 (1996) de Francine Christophe; La haine de la famille (2001) de Catherine Cusset; Autobiographie de personne (1999), Fin E Suite (2001) et Petite biographie pour un rêve (2003), las tres obras de Esther Orner; J'ai pas pleuré (2002) de Ida Grinspan; Survivre et vivre (2004) de Denise Epstein; Un paysage de cendres (2006) de Elisabeth Gille; Le

20. En este trabajo solo citaremos algunas obras propias de la literatura del Holocausto de escritoras francesas y las obras de la escritora israelí de expresión francesa, Esther Orner. Para una periodización de los relatos testimoniales de las escritoras judías de la Shoá, remitimos al estudio «Los relatos testimoniales de mujeres judías durante la Shoá. Estado de la cuestión entre el final de la guerra y el final de siglo» realizado por José Luis Arráez en el presente ensayo.

21. Considerando como tales a las escritoras nacidas antes y durante la guerra.

22. Considerando como tales a las escritoras nacidas tras la guerra.

23. Régine Waintrater concluye este tercer tiempo en 2003 coincidiendo con la fecha de publicación de su libro Sortir du génocide. En nuestro caso, lo hacemos coincidir con la época actual.

Feminismo/s 34, diciembre 2019, pp. 175-198 
remplaçant (2009) de Agnès Desarthe Ma vie balagan (2008), Et tu n'es pas revenu (2015) et L'amour après (2017) de Marceline Loridan-Yvens.

Las publicaciones correspondientes al tercer periodo serían mucho más amplias, pues en este caso coinciden testimonios correspondientes tanto a las primeras generaciones de la memoria de la Shoá como a las generaciones posteriores tras la memoria de la Shoá.

\section{ESCRITURA TRANSGENERACIONAL Y PERSPECTIVA DE GÉNERO}

Progresivamente, las y los supervivientes van desapareciendo y sus testimonios sólo podrán ser escuchados por medio de vídeos, grabaciones o ser leídos. El tercer periodo de la literatura de la Shoá da una clara muestra de hacia dónde se dirige este tipo de literatura, con sus luces y sus sombras. Durante ese periodo, las publicaciones entre las distintas generaciones de la Shoá confluyen. Es el caso de la escritura de Vivianne Forrester ${ }^{24}$, pues sus obras abarcan los dos periodos mencionados. Tomando como referencia la escritura de Vivianne Forrester, Sarah Kofman, Francine Christophe y Catherine Cusset, podemos señalar las siguientes características propias de la escritura transgeneracional desde la perspectiva de género: del ensayo y la ficción a la escritura del yo y las relaciones madres e hijas.

\subsection{Del ensayo y la ficción a la escritura del yo: una cuestión de géneros literarios}

Forrester escapó del nazismo huyendo a España con su familia, en 1943. Sus primeras obras, ficciones, sobre las persecuciones nazis, datan de 1970. En Loeil de la nuit narra las viviencias de dos hermanas que tras la guerra se reencuentran, pero ya nada volverá a ser lo mismo. Para Forrester, como para otras escritoras, la ficción se convierte, en un primer momento, en una manera de salir de ese silencio donde poder narrar lo indecible sin que exista una clara relación entre el yo que escribe y el que se escribe. Vivianne Forrester, al igual que Sarah Kofman, tardó años en publicar su primer relato autobiográfico Ce soir, après la guerre (1992), donde narra el periplo que sufrió

24. Nació en París en 1929 y falleció en 2013.

Feminismo/s 34, diciembre 2019, pp. 175-198 
junto a su familia para poder llegar a España. De este relato se desprenden preguntas trascendentales: «Qu'ai-je appris de la guerre?»o «Qu'ai-je appris de la guerre, pendant l'Occupation?» (Forrester 10-11), y una respuesta clara y contundente: «Je me souviens de moi» (Forrester 10). Y ese «moi», es el «Je» que se construye también por medio de la escritura.

Como en el caso de Vivianne Forrester, a Sarah Kofman sólo el tiempo le concederá otra manera de narrar, y el yo que escribe en sus ensayos filosóficos pasará a ser un yo en primera persona. Al fin, la escritura será una escritura autobiográfica, aunque solo sea como presagio de un final trágico, pues con la publicación de Rue Ordener, Rue Labat (1994) se cumple la profecía, aquella que da por única y última vez para luego desaparecer con el tiempo. Kofman respondió a la eterna pregunta que sobrevuela la escritura transgeneracional de la Shoá: «¿Qué heredamos de nuestros padres?». De él, solo me queda un bolígrafo, dijo Kofman al inicio de su relato autobiográfico. En efecto, un bolígrafo como metonimia de la escritura que, al fin, trasciende los muros del silencio: «Parce qu'il était juif, mon père est mort à Auschwitz: comment ne pas le dire? Et comment le dire? Comment parler de ce devant quoi cesse toute possibilité de parler?» (Kofman, Paroles suffoquées 15-16). Así lo expresaba Sarah Kofman, unos años antes, en 1987, en el ensayo Paroles suffoquées.

\subsection{Las relaciones madres e hijas: del silencio a la palabra}

Otra de las características que podemos destacar en este tipo de escritura viene marcada por una de las líneas de investigación desarrolladas desde una perspectiva de género. Se trata de la relación entre madres e hijas que, en el caso de la literatura de la Shoá perteneciente al tercer tiempo, se convierte en uno de los temas centrales.

En las figuras maternas recae la responsabilidad de explicar, ante las miradas atónitas de las hijas, lo que es inexplicable, es decir, qué sucede. ¿Por qué esa persecución sistemática? ¿Por qué hay que huir? Así lo expresaba la escritora Francine Christophe en su autobiografía Une petite fille privilégiée. Une enfant dans le monde des camps 1942-1945:

Maman a peur. J'ai peur. Partout, tout le temps, les gens à étoile disparaissent sans retour [...] J'ai appris que je suis juive, que je suis un monstre, et que je dois me cacher. J'ai peur tout le temps (23). 
Para la niña resulta difícil comprender qué ha podido suceder para que su madre cambiara: «Maman, quand je la regarde bien, je crois qu'on me l'a changée» (94-95). O en ese mismo sentido, podemos destacar la magnífica descripción que nos ofrece Vivianne Forrester en Ce soir, après la guerre:

Je me souviens d'un jour où les palmiers dégoulinaient de pluie. On ne distinguait plus le ciel de la mer [...] Je découvrais ma mère, assise sur une chaise, seule au milieu du salon. Elle regardait ses pieds [...] «Maman, qu'est-ce que tu as ?» Et sa voix très basse, l'ombre de sa voix : «Rien. Il pleut. Je suis juive» (75).

El silencio y la incomprensión van invadiendo progresivamente estas relaciones. En numerosas ocasiones, debido a una obligada separación, las niñas son incapaces de comprender lo que sucede, pues para ellas se trata de un abandono. Viven una separación traumática de la que tardarán años en recuperarse, aunque nunca se podrá franquear completamente el muro de la ausencia. Sarah Kofman es un claro ejemplo de esa separación. Siendo muy niña y tras el arresto de su padre, su madre para protegerla se ve obligada a cederla a otra mujer, mientras ella permanece escondida. Para la niña, incapaz de entenderlo, la separación de su madre es vivida de manera intensa y dolorosa. Para ella, cuando era muy pequeña, el único peligro existente era la separación de su madre: «le vrai danger: être séparée de ma mère» (Kofman, Rue Ordener, Rue Labat 33). Al llegar la guerra y mientras se iban incrementando las detenciones masivas: «ma mère eut peur de me garder auprès d'elle. Elle fit de nouvelles tentatives pour me cacher» (Kofman, Rue Ordener, Rue Labat 37). Y así, entre dos calles parisinas (Rue Ordener, Rue Labat) separadas por una parada de metro, y con dos madres, la madre biológica y la madre de acogida, la pequeña Sarah conseguirá escapar a la guerra. Sin embargo, la escisión entre madre e hija será ya inevitable. Los celos entre la madre biológica y la madre de acogida se convierten en una realidad, y cuando se produce nuevamente el momento de la separación de la madre de acogida, el dolor se vuelve intolerable:

Au cinquième étage de la rue Labat, ma mère, accompagnée de deux hommes, était sur le palier: ils m'arrachèrent violemment à mémé, me portèrent dans leurs bras jusque dans la rue. Ma mère me frappait en hurlant en yiddish: «Je suis ta mère! je suis ta mère! je me fiche de ce qu'a décrété le tribunal, tu m'appartiens!» (Kofman, Rue Ordener, Rue Labat 71). 
Otro ejemplo de relación conflictiva lo podemos encontrar en La haine de la famille (2001) de Catherine Cusset. Perteneciente también al tercer tiempo, Catherine Cusset formaría parte de esa generación, en este caso tercera generación, nacida tras la memoria de la Shoá. Es una generación cuya escritura ya no viene marcada por la necesidad de salir de silencio y de narrar una experiencia traumática, pues no han vivido ni los campos, ni la guerra, pero sí han sido víctimas del silencio que ha rodeado a la familia. Por lo tanto, ya no se trata de abordar la Shoá de manera directa sino de forma transversal, como sucede en La haine de la famille de Cusset. Para Cusset, por medio de la creación del personaje femenino de Marie, alter ego de sí misma, intenta comprender qué tipo de relación une o desune a su familia, y de forma más concreta la lleva a enfrentarse con la figura materna. Para ello, recuerda un episodio de la vida de su madre del que ella no fue testigo pero que marcó tanto a su madre como a su abuela para siempre, y que por lo tanto daría una explicación a esa «haine de la famille». Su madre es judía. En 1943, la madre de la narradora es testigo del intento de detención de su propia madre, es decir de la abuela de la narradora, y de cómo esta consiguió salvar a sus hijas.

Nous avons l'ordre de vous arrêter, vous et vos filles -Certainement pas mes filles [...] Il y a une erreur leur père est aryen -mais vous, vous êtes juives. Mais leur père est aryen, je vous dis et prisonnier de guerre. Vous ne les emmènerez pas. De plus elles sont baptisées (Cusset 189).

Y así, un día, al fin, la madre de la narradora consigue salir de su silencio y expresar el miedo que sintió cuando vio cómo su madre era arrestada: «Tu comprends comment ça a pu conditionner toute ma relation à ma mère! Pourquoi je n'ai jamais pu la quitter! J'avais peur dès que j'étais loin d'elle. Je n'ai plus cessé d'avoir peur» (Cusset 195). Sin embargo, entre la narradora y la madre, surge una figura intermedia creada por la hija, fruto de la relación tormentosa con la madre: la figura del nazi, cuando afirma lo siguiente: «Le nazi, c'est moi. Moi qui n'aime pas ma mère» (Cusset 216).

Así, con una frase tan lapidaria como la pronunciada por Marie en La haine de la famille, queremos ir concluyendo este apartado, conscientes de que todavía queda mucho trabajo por hacer en este tipo de escritura transgeneracional desde una perspectiva de género. Se trataría, por un lado, de seguir dando visibilidad a las escritoras de este último periodo, pues si bien ya son pocas las escritoras supervivientes que puedan publicar debido a su 
progresiva desaparición por cuestiones de edad, el camino emprendido por aquellas que descubren, tras años de silencio familiar, la pérdida de un algún familiar, o incluso descubren sus orígenes judíos, supone un conflicto identitario que implica una necesidad de ser narrado. Y, por otro lado, debido precisamente a ese conflicto, se hace necesario abordar la Shoá desde otra vertiente, transversal pero no por ello inexistente.

\section{CONCLUSIÓN}

Iniciamos este recorrido con una pregunta esencial, y cual frase leitmotiv nos ha guiado a lo largo de esta investigación: ¿puede la literatura transgeneracional de la Shoá ser estudiada desde una perspectiva de género?

Tras acercarnos a los estudios de la Shoá y a los estudios de género desde una perspectiva de género, hemos podido observar que, a pesar de sus discrepancias y de los debates suscitados a lo largo de los años, sigue siendo vital que la literatura de la Shoá y los estudios de género se complementen.

Es necesario también ir más allá de la simple clasificación establecida que distingue a las primeras, segundas y en menor medida a las terceras y cuartas generaciones en la literatura de la Shoá. Por ello, abogamos por una distinción que sea más precisa como la propuesta: distinguir entre las obras narrativas pertenecientes a la primera generación de la memoria de la Shoá de las obras narrativas pertenecientes a la segunda generación tras la memoria de la Shoá. Así, podrán ser abordadas con sus similitudes (necesidad de salir del silencio) y sus diferencias (las relaciones entre madres e hijas, el conflicto identitario).

Tras este breve recorrido, podemos afirmar que la literatura transgeneracional de la Shoá debe también ser abordada desde una perspectiva de género, pero sin olvidar que todavía existe un largo camino por recorrer. Actualmente, es importante reflexionar y analizar los textos literarios de estas escritoras para desentrañar la belleza de las voces que se escriben y se dejan leer.

\section{REFERENCIAS BIBLIOGRÁFICAS}

Arráez, José Luis, y Amelia Peral. Memoria de la Shoá. Literatura y testimonio. Madrid: Sefarad Editores, 2012.

Azouvi, François. Le mythe du grand silence. Auschwitz, les Français, la mémoire. Paris: Fayard, 2012. 
Azouvi, François. Le mythe du grand silence. Auschwitz, les Français, la mémoire. Paris: Folio-Gallimard, 2015.

Bensoussan, Georges. Histoire de la Shoah. Paris: PUF, 2007.

Bock, Gisela. Genozid und Geschlecht. Jüdische Frauen im nationalsozialistischen Lagersystem. Frankfurt am Main: Campus Verlag GmbH, 2005.

Bridenthal, Renate, Atina Grossman, y Marion Kaplan. When Biology became Destiny. Women in Weimar and Nazi Germany. New York: Monthly Review Press, 1984.

Camarero, Jesús (eds), Le silence dans l'écriture de la Shoah. Çedille 5 (2015).

Christophe, Francine. Une petite fille privilégiée. Une enfant dans le monde des camps 1942-1945. Paris: Gallimard, 1996.

Cusset, Catherine. La haine de la famille. Paris: Gallimard, 2001.

Cushman, Sarah. The Women of Birkenau. Worcester MA: Clark University, 2010.

Dayan Rosenman, Anny. Les alphabets de la Shoah. Survivre. Témoigner. Ecrire.

Paris: CNRS, 2007.

Distel, Barbara. Frauen im Holocaust. Gerlingen: Bleicher Verlag, 2001.

Distel, Barbara, y Wolfgang Benz. Der Ort des terrors. Verlag Dachauer Hefte: Metropol Verlag, 2006.

Ernot, Isabelle. «Women and/in the Holocaust»: à la croisée des Women's-Gender et Holocaust Studies (Années 1980-2010)». Genre E Histoire 15 (2014-15).

Ertel, Rachel. «La littérature e(s)t le lien». Des témoins aux héritiers. L'écriture de la Shoah et la culture européenne. Eds. Luba Jurgenson y Alexandre Prstojevic. Paris: Pétra, 2012.

Forrester, Viviane. L'oeil de la nuit. Paris: Grasset, 1970.

Forrester, Viviane. Ce soir, après la guerre. Paris: Fayard, 1992.

Goldenberg, Mirna, y Elizabeth Baer. Experience and Expression: Women, the Nazis, and the Holocaust. Detroit: Wayne State University Press, 2003.

Goldenberg, Mirna. Before All Memory is Lost: Women's Voices from the Holocaust. Toronto: The Azrieli Foundation, 2017.

Grinspan, Ida, y Bertrand Poirot-Delpech. J'ai pas pleuré. Paris: Robert Laffont, 2002.

Grisnpan, Ida. Yo no lloré. Barcelona: Anthropos, 2010.

Kandel, Liliane. «Une pensée empêchée: des usages du «genre», et de quelques-unes de ses limites». Les Temps Modernes 587 (1996): 220-248.

Kandel, Liliane. «Sur quelques lectures féministes du nazisme». 1939-1945: combats de femmes. Françaises et Allemandes, les oubliées de l'histoire. Éd. Évelyne Morin-Rotureau. Paris: Autrement, 2001. 111-121. 
Kandel, Liliane (dir.). Féminismes et nazisme. Paris: Odile Jacob, 2004.

Kandel, Liliane. "On ne naît pas innocent(e), on le devient». Féminismes et nazisme. Paris: Odile Jacob, 2004. 8-30.

Katz, Esther, y Joan Ringelheim. Proceedings of the Conference: Women Surviving the Holocaust. New York: The Institute for Research in History, 1983.

Kofman, Sarah. Paroles suffoquées. Paris: Galilée, 1987.

Kofman, Sarah. Rue Ordener, Rue Labat. Paris: Galilée, 1994.

Langfus, Anna. Le sel et le soufre. Paris: Gallimard, 1960.

Langfus, Anna. Les bagages de sables. Paris: Gallimard, 1962.

Langfus, Anna. Saute Barbara. Paris: Gallimard, 1965.

Lapierre, Nicole. «Le cadre référentielle de la Shoah». Ethnologie française XXXVII/3 (2007): 475-482.

Levi, Primo. Si questo è un uomo. Turín: Giulio Einaudi, 1947.

Loridan-Yvens, Marceline. Ma vie balagan. París: Robert Laffont, 2008.

Loridan-Yvens, Marceline. Et tu n'es pas revenu. París: Grasset, 2015.

Loridan-Yvens, Marceline. L'amour après. París: Grasset, 2017.

Ofer, Dalia, y Leonore J. Weitzman (eds). Women and the Holocaust. New Haven and London: Yale University Press, 1998.

Ofer, Dalia, y Leonore J. Weitzman. «Sur la spécificité de la place des femmes dans la Shoah. Fondements théoriques d'une analyse». Revue d'Histoire de la Shoah 188 (2008): 479-506.

Peral, Amelia. «En cada mujer dormita una madre. Entrevista a Ida Grinspan, superviviente de Auschwitz». Memoria de la Shoá. Literatura y testimonio. José Luis Arráez y Amelia, Peral (coords). Madrid: Sefarad Editores, 2012. 279-314.

Peral, Amelia. «Una infancia robada. Entrevista a Víctor Pérahia, superviviente de varios campos de concentración». Memoria de la Shoá. Literatura y testimonio. José Luis Arráez y Amelia, Peral (coords). Madrid: Sefarad Editores, 2012. 315-328.

Peral, Amelia. «Au-delà des mères perdues ou comment nommer la mère après la Shoah», Confluences (2012): 128-142.

Peral, Amelia. «Le silence, ce cri qui résonne dans l'écriture de Viviane Forrester», Çédille 5 (2015): 137-157.

Potel, Jean-Yves. «La Pologne d'Anna Langfus». Bulletin du Centre de recherche français à Jérusalem [En ligne] 22 (2011). Mis en ligne le 25 mars 2012. 28 julio 2019. <http://journals.openedition.org/bcrfj/6583> 
Potel, Jean-Yves. Les disparitions d'Anna Langfus. Paris: Editions Noir sur Blanc, 2014.

Prstojevic, Alexandre. «La question de la périodisation». Des témoins aux héritiers. L'écriture de la Shoah et la culture européenne. Dirs. Luba Jurgenson y Alexandre Prstojevic. Paris: Petra, 2012: 17-31.

Steinecke, Hartmut. «La Shoah dans la littérature de la 'deuxième génération'». Revue d'Histoire de la Shoah 201 (2014): 239-264.

Rawicz, Piotr. Le sang du ciel. Paris: Gallimard, 1961.

Ringelheim, Joan. Women and the Holocaust: A Reconsideration of Research». Signs 15.4 (1985): 741-761.

Thalmann, Rita. Être femme sous le III ${ }^{e}$ Reich. Paris: Laffont, 1982.

Thalmann, Rita. Tout commença à Nuremberg: entre histoire et mémoire. Paris: Berg International, 2004.

Viart, Dominique, y Bruno Vercier. La littérature française au présent. Héritage, modernité, mutations. Paris: Bordas, 2005.

Waintrater, Régine. Sortir du génocide. Témoigner pour réapprendre à vivre. Paris: Payot, 2003.

Waxman, Zoe. Women in the Holocaust: A Feminist History. Oxford: Oxford University Press, 2017.

Wiesel, Elie. La nuit. Paris: Éditions de Minuits, 1958.

Wieviorka, Annette. Auschwitz, la mémoire d'un lieu. Paris: Hachette, 2005.

Wieviorka, Annette. «Préface». Les alphabets de la Shoah. Annie Dayan Rosenman. Paris: CNRS, 2007.

\section{Sitios web}

Mémorial de la Shoah. Musée, Centre de documentation.15 julio 2019. $<$ http://www.memorialdelashoah.org/>

Yad Vashem. The World Holocaust Remembrance Center. 15 julio 2019. <https://www.yadvashem.org/>

Museums Portal Berlin. 15 julio 2019. <https://www.museumsportal-berlin.de/en/museums/denkmal-fur-dieermordeten-juden-europas-ort-der-information/>

United State Holocaust Memorial Museum. 15 julio 2019. $<$ https://www.ushmm.org/es> 